\title{
Rib-reinforced Shell Structure
}

\author{
Wei $\mathrm{Li}^{1}$, Anzong Zheng ${ }^{2}$, Lihua You $^{2}$, Xiaosong Yang ${ }^{2}$, Jianjun Zhang ${ }^{2}$ and Ligang Liu ${ }^{\dagger 1}$ \\ ${ }^{1}$ University of Science and Technology of China, China $\quad{ }^{2}$ Bournemouth University, UK
}

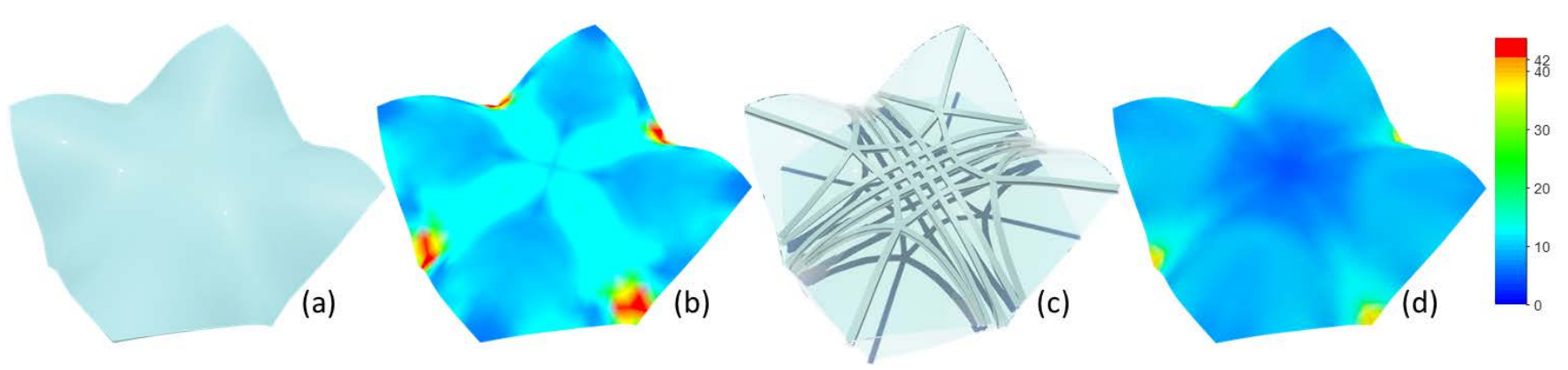

Figure 1: We present a computational framework to generate ribs to enhance the structural strength and stiffness of a shell (a). Our ribreinforced shell structure (c) costs the same amount of material as a pure shell without any ribs (a), while achieving much better mechanical performance. And (b) and (d) are coloring visualizations of the magnitude of von Mises stress of (a) and (c), respectively, under the same load and boundary conditions. Warmer color represents higher stress and red encodes stress exceeding the yield strength $42 \mathrm{MPa}$. The maximum stress in (b) is $109.84 \mathrm{MPa}$, which far exceeds $42 \mathrm{MPa}$, while the maximum stress in (d) is below $40 \mathrm{MPa}$ with the contribution from ribs. The maximum stress of the shell is about 3 times of the stress of the rib-reinforced shell structure, indicating the latter achieves much higher strength than the former. We also fabricated the two structures by 3D printing and made a stiffness comparison as shown in Figure 19 which indicates much higher stiffness of the rib-reinforced shell structure.

\begin{abstract}
Shell structures are extensively used in engineering due to their efficient load-carrying capacity relative to material volume. However, large-span shells require additional supporting structures to strengthen fragile regions. The problem of designing optimal stiffeners is therefore becoming a major challenge for shell applications. To address it, we propose a computational framework to design and optimize rib layout on arbitrary shell to improve the overall structural stiffness and mechanical performance. The essential of our method is to place ribs along the principal stress lines which reflect paths of material continuity and indicates trajectories of internal forces. Given a surface and user-specified external loads, we perform a Finite Element Analysis. Using the resulting principal stress field, we generate a quad-mesh whose edges align with this cross field. Then we extract an initial rib network from the quad-mesh. After simplifying rib network by removing ribs with little contribution, we perform a rib flow optimization which allows ribs to swing on surface to further adjust rib distribution. Finally, we optimize rib cross-section to maximally reduce material usage while achieving certain structural stiffness requirements. We demonstrate that our rib-reinforced shell structures achieve good static performances. And experimental results by $3 D$ printed objects show the effectiveness of our method.
\end{abstract}

Categories and Subject Descriptors (according to ACM CCS): I.3.5 [Computer Graphics]: Computational Geometry and Object Modeling-Curve, surface, solid and object representations.

Keywords: Architectural geometry, Rib-shell structure, Principal stress

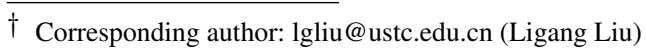

\section{Introduction}

Shell structures, such as aircraft fuselages, boat hulls, and largespan roofs, are lightweight constructions using shell elements. And 

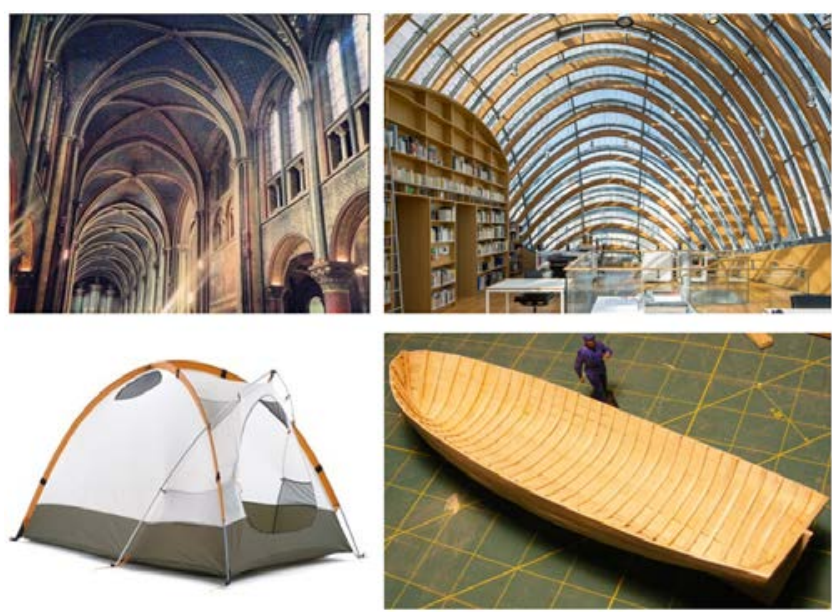

Figure 2: Various forms of rib-reinforced shell structures. Top left: rib vaulting in the nave of Reims Cathedral. Top right: transparent bubble dome with ribs of larch. Bottom left: a tent. Bottom right: a boat.

these elements, typically curved, are assembled to make large structures. Shell structures achieve high degree of reserved strength and structural integrity, and greatly release the internal space [VK01]. However, an accompanying dilemma is weakened stiffness and strength when a shell has a large span and/or thin thickness. Hence in most cases, additional supports or stiffeners like pillars [EDE10], scaffoldings [DB11], frames [WWY*13], or ribs [LS03], are introduced to reinforce the structural performance and mechanical property. Among these, ribs have unique advantages and are widely used in engineering as shown in Figure 2.

With ribs closely attached to one or both sides of shell, ribreinforced shell structures have many good mechanical and geometrical properties. First of all, ribs don't occupy too much internal space compared with pillars, scaffoldings or frames. Secondly, ribs utilize the bending characteristics, so they avoid stress concentration and have better stability [ARR12, CK13], while pillars, scaffoldings and frames may cause buckling under different loads. Finally, ribs can have variable cross-sectional shapes which achieve different performance and meet kind of goals [LS03]. In engineering, most works focus on the analysis of rib-shell structure, namely, analyze the mechanical property given the layout of ribs [PS01, ARR12]. Only a small number of works involve the location of ribs on some simple surfaces like plates, cylinders, etc [LS03, DJMH13]. And in computer graphics, researchers pay more attention to the generation and optimization of triangle-based, quad-based or hex-dominant grid-shell structures [LXW*11, CW07, LLW15]. And they mainly take geometry into account, and only a few consider the static performance [PTP* $15, \mathrm{SB} 10]$. Although there are many existing related works, no computational method to construct and optimize rib layout on shell with arbitrary shape is proposed so far.

Our goal is to place ribs on a shell to best enhance its overall mechanical performance. However, the construction of rib-reinforced shell structures is a very challenging problem. The difficulties lie in three parts, namely,

- how many ribs to use,

- where to place, and

- what's an effective cross-section?

To this end, we propose a computational framework to design and optimize rib layout on arbitrary surface. The basic idea of our method is to place ribs along the principal stress lines which can approximately best reinforce the stiffness of a shell [KLC16, TFCM16]. Our system first generates a dense rib network, followed by a simplification procedure which removes redundant ribs with little contribution. Then we perform a rib flow optimization to readjust the distribution of ribs and a size optimization to achieve certain stiffness requirement with least amount of material. Finally, the cross-sectional shape of ribs is optimized by using T-section to further reduce material usage while maintaining overall physical properties.

In particular, our contributions can be summarized as follows.

- We provide a computational framework to generate and optimize rib network to reinforce the structural stiffness and physical performance of shells.

- We propose a rib flow optimization which takes each rib as a whole and allows ribs swing on surface, to adjust rib layout to achieve better structural performance.

To the best of our knowledge, this work proposes the first computational method to reinforce arbitrary shell by generating and optimizing ribs on them. Experimental results have shown that our generated rib-shells are feasible and cost-effective lightweight structures for reinforcing various spatial structures.

\section{Related Works}

Architectural Geometry In the field of architectural geometry, most contributions are concerned with the optimization of geometric properties of polygonal meshes, mostly quadrilateral mesh to approximate a free-form surface. Many works address the planarity of faces, such as the construction of PQ (planar quad) meshes [LXW*11, YYPM11,ZSW10], CP (cell packing) meshes [SHW$\left.\mathrm{P} 09, \mathrm{PJH}^{*} 15\right]$, and polygonal or hexadecimal dominant meshes [TSG*14, CW07, LLW15]. Some works try to approximate surface by a union of patches, so-called panels [PSB $\left.{ }^{*} 08, \mathrm{EKS}^{*} 10\right]$. Among these only a few works take the statics of structures into account when designing the grid structure. Pietroni et al. introduced a framework for the generation of grid-shell structures that achieve excellent static performances based on Voronoi diagram$\mathrm{s}$ [PTP* 15]. Schiftner et al. presented a simple but effective staticsaware initialization procedure for the layout of PQ meshes approximating a given freeform surface [SB10]. Our work focuses on the generation and optimization of rib network that is attached on the surface of shell, to enhance the overall physical performance.

Optimization of Lightweight Structure Recently, the optimization problem of lightweight structures to save materials and enhance mechanical performance draws more and more interests from researchers. Wang et al. [WWY*13] introduced skin-frame structure which replaces the solid interior of an object by truss scaffoldings. The structure is optimized iteratively to reduce the truss while 
accounting for several physical and geometrical constraints. Lu et al. [LSZ* 14] proposed honeycomb-like structure by hollowing object with global optimization of the strength-to-weight ratio. Zhao et al. [ZXZ*16] proposed a method to optimize the thickness of shell to improve its physical property. Zhou et al. [ZKWG16] introduced a generic and extensible method that directly optimizes a shape subject to physical and geometric constraints. [WAWS17] presented a method to generate bone-like porous structures as lightweight infill that maintain good structural performance. Unlike the above, our method strengthens objects by adding ribs attached to the surface, which saves lots of inner space and avoids stress concentration.

Rib-shell Structure There are lots of researches on rib-stiffened shell structure in engineering. Most works focus on the analysis of a given rib-shell structure [Joh58, PS01, ARR12, CK13], namely investigating the stiffening effect of ribs on a certain surface under given external load. Others consider the optimization of size, shape or location of ribs on surface to enhance the performance of total structure [LS14, WAZ17, ACTR04]. And only a few works study the design of ribs on given shell. Lam et al. introduced an automatic method to place ribs on surface by optimizing the thickness of shell first, then put ribs on surface where the thickness exceeds a given threshold value [LS03]. Another method to design ribs is the so-called adaptive growth technique. Stiffeners start from the seed points, grow and branch off towards direction with the maximal effectiveness of the global mechanical performance, which is dependent on the design sensitivity of the current structure [DJMH13,JDX14]. Chung and Lee [CL97] studied the optimal shapes and locations of ribs in order to increase the stiffness of structures using the topology optimization technique. Szczepanik et al. proposed to use particle swarm algorithm to optimize rib locations [SB12]. But almost all of these works just consider some very simple surface. And for an arbitrary 3D freeform surface, the design of rib-shell structure is still a tough job. We provide a computational framework to generate and optimize ribs on arbitrary surface for the first time.

Principal Stress Lines Inspired Structure Optimization Principal stress lines are pairs of orthogonal curves that indicate trajectories of internal forces [TM15]. There are some works on structure optimization that inspired by principal stress lines. Su et al. [SZXG14] presented an algorithm to generate grid that are concordant with the force flow. Kwok et al. [KLC16] provided a novel growth method based on principal stress lines to define the geometry and topology of structure. Tam et al. [TFCM16] introduced stress line additive manufacturing which can achieve better structural performance. Wu et al. [WAWS17] found that microstructures of trabecular bone are aligned along the principle stress directions and proposed an approach to generate bone-like porous structures. Our rib-shell structure is also inspired by principal stress lines. But we aim at placing ribs on the surface that follow the principal stress lines.

\section{Overview}

We provide users a framework to create rib network attached to shell to enhance its structural performance. As shown in Figure 3, the framework mainly comprises four stages: 1) rib network initial- ization, 2) rib network simplification, 3) rib flow optimization and 4) rib cross-section optimization.

Rib Network Initialization Given a surface, we regard it as a shell by assigning it a thickness. Then we impose external load and perform Finite Element Analysis (FEA) on the shell. From the FEA results, we compute the principal stress field on the surface, which shows desirable material continuity and encodes the optimal topology [TM15]. We aim at placing ribs along the two orthogonal directions. Using this stress field, we calculate a global parametrization, and then generate a quad-mesh whose edges align with these directions. From the quad-mesh, we extract a rib network which consists of tree-like, circular and curved rib elements.

Rib Network Simplification We already have a dense rib network, but in fact, perhaps not all the ribs are needed. Some ribs may have little contribution to the shell. Therefore we simplify the rib network by dropping some rib elements according to their contribution which is determined by the strain energy increment when adding a rib element.

Rib Flow Optimization In the previous stage, we simplify rib network by just removing some rib elements. However, this removal operation affects the remaining ribs. A further adjustment on the layout of remaining ribs is needed to achieve better distribution. We formulate a rib flow optimization to allow ribs swing on surface and reduce the total strain energy with limited material usage.

Rib Cross-section Optimization After the rib flow optimization, the topology of rib network is determined. We pursue to optimize the rib cross-section with the goal of satisfying certain physical strength requirement with least material usage. Considering the good mechanical property and extensive use in practical engineering of T-section, we use this type of shape for our ribs. We first assume the cross-section of ribs is rectangular, and optimize the width and height of ribs to minimize the material usage while preserving the structural stiffness and performance of rib-shell structure. Then we optimize the cross-sectional shape of ribs using hyperelliptic T-shaped cross-section and find the optimal configuration.

\section{Rib-shell Structure}

\subsection{Rib-shell Structure}

Given an input mesh $\mathcal{M}^{0}$, our rib-shell structure $\mathcal{H}$ consists of a thin shell $\mathcal{S}$ and ribs $\mathcal{R}$, as shown in Figure 4. $\mathcal{S}$ is a thin shell between $\mathcal{M}^{0}$ and its offset $\mathcal{M}^{1}$ with a thickness $t$. $\mathcal{R}$ is composed of a set of ribs $\mathcal{R}=\left\{R_{i}, i=1,2, \cdots, n\right\}$. Each rib is closely attached to one or both sides of $\mathcal{S}$ and contains a set of consecutive rib points $R_{i}=\left\{R_{i j}, j=1,2, \cdots, n_{i}\right\}$, where the subscript $i$ indicates the $i^{t h}$ rib, and $i j$ indicates the $j^{\text {th }}$ node of the $i^{\text {th }}$ rib.

\subsection{Rib Network}

All the ribs $\mathcal{R}$ in a rib-shell structure compose a rib network $\mathcal{N}$ which contains three fundamental element sets, i.e., curved $\mathcal{R}^{c}$, 


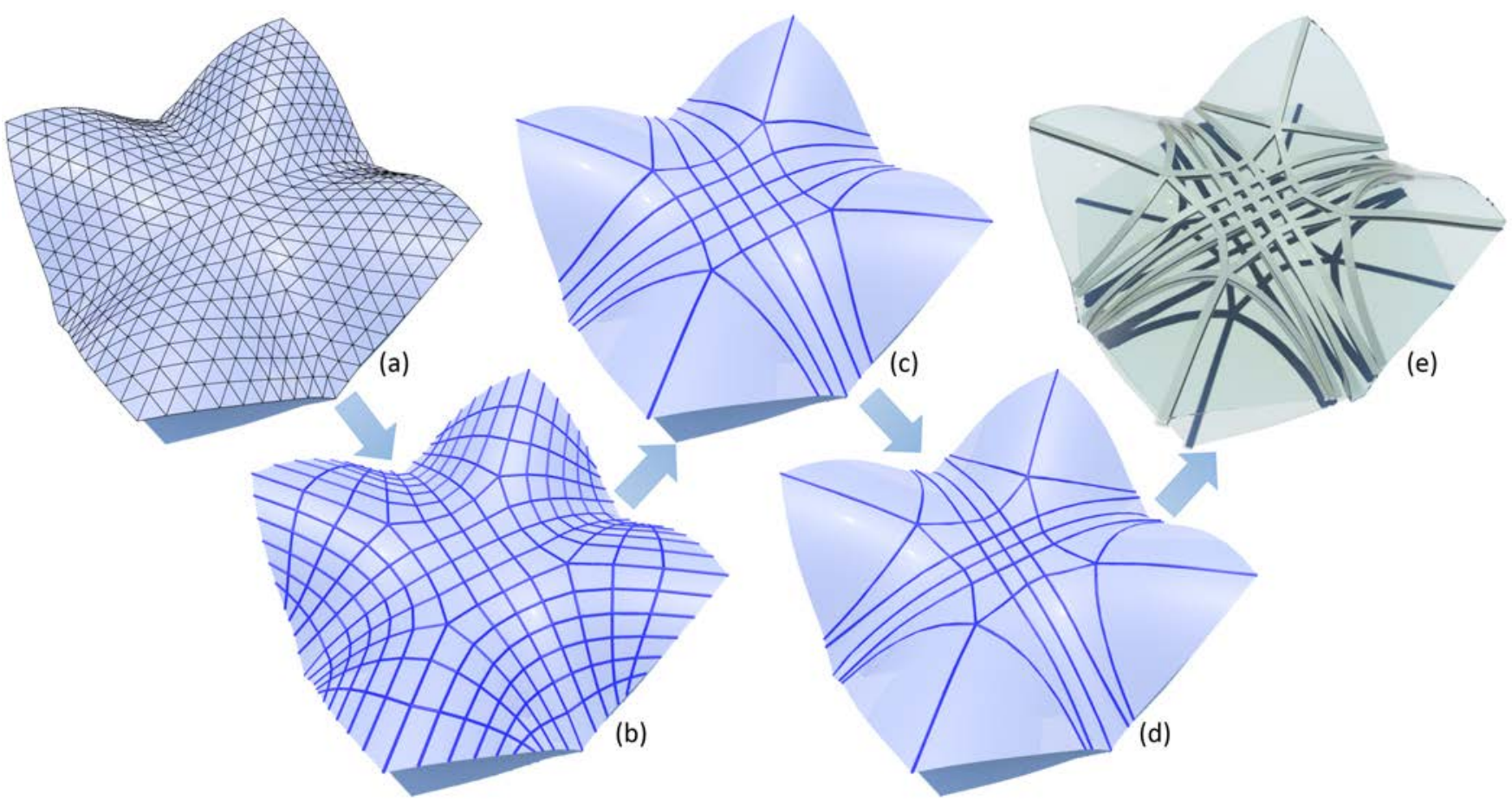

Figure 3: System overview: Given an input surface (a), we first generate a dense rib network (b), followed by a simplification process to remove the ribs with little contribution (c). Then we perform a rib flow optimization to adjust the layout of ribs (d). Finally we optimize the cross-section of ribs using hyperelliptic T-section (e).

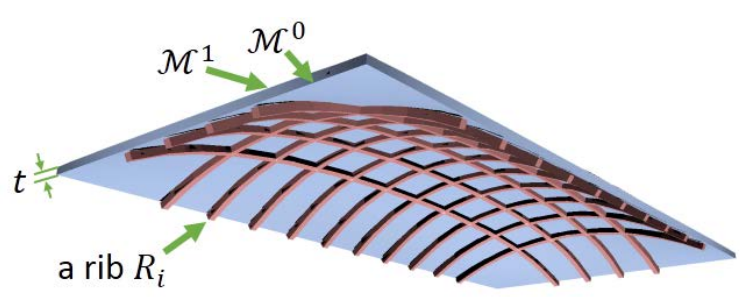

Figure 4: Illustration of a rib-shell structure $\mathcal{H}$ consists of a thin shell $\mathcal{S}$ (in blue) with a thickness $t$ and a network of ribs $\mathcal{R}$ (in brown).

$\underline{\text { circular }} \mathcal{R}^{o}$ and tree-like $\mathcal{R}^{t}$ rib element sets. A rib element consists of ribs and has semantic information. Usually the tree-like rib element plays a more important role in reinforcing a shell. And circular and curved rib elements enhance local stiffness and strength and assist to increase global performance of rib-shell structures. Figure 5 shows the three types of rib elements in a rib network. Note that curved rib element is the fundamental rib element which is also called rib for convenience. A tree-like rib element consists of several curved rib elements, and a circular rib element can be regarded as a curved rib element whose two ends meet at a same point.

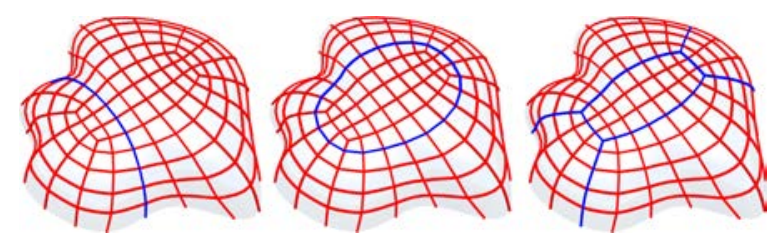

Figure 5: From left to right: curved, circular and tree-like rib elements. The right tree-like rib element consists of eight curved ribs.

\subsection{Stress Computation}

The goal of stress computation is to set up the static equilibrium equation of rib-reinforced shells to calculate the nodal displacements using Finite Element Method (FEM). Since our rib-shell structure is a coupled construction of shell and ribs with each rib closely attached to the surface of shell (Figure 6), static analysis should take them as a whole. Before performing FEA on the coupled structure, we remesh the surface such that all the ribs lie on the edges of the resultant triangular mesh. To simulate the thin shell, we adopt Constant Strain Triangular (CST) element [ABVW14] for membrane deformation and Discrete Kirchhoff Triangular (DKT) element [VK01] for bending behavior. Each rib is discretized according to the edges of the triangular mesh and modeled as beam elements, and the two ends of each beam have corresponding mesh vertices. The contribution of the ribs to the shell is obtained by superimposing the element stiffness matrix $\mathbf{K}_{e}^{r}$ of the ribs to the el- 
ement stiffness matrix $\mathbf{K}_{e}^{s}$ of the shell as shown in Figure 6. In the figure, the superscripts $s$ and $r$ indicate the shell and the ribs, respectively, and the subscripts $i, j$ and $k$ indicate the three nodes of a triangular finite element among which $j$ and $k$ are also the two nodes of a beam finite element. After we get the assembled stiffness
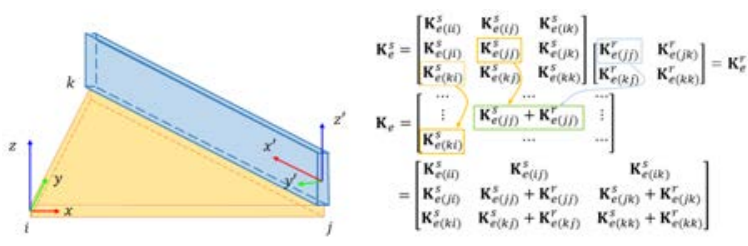

Figure 6: Stiffness matrix assembling for a coupled structure.

matrix K. The node displacements can be obtained by solving the following equilibrium equation

$$
\mathbf{K u}=\mathbf{f},
$$

where $\mathbf{f}$ is the vector of node forces, $\mathbf{u}$ denotes the vector of resulting node displacements. We validate the accuracy of stress computation for the couple system by comparing the results with ABAQUS which is a well-known commercial FEM software.

Von Mises Stress The von Mises stress is widely used to predict yielding of materials under any loading condition [ $\log 11]$. A material is said to start yielding when its von Mises stress reaches a critical value known as the yield strength. We thus use von Mises stress in our system, which is a scalar deduced from the Cauchy stress tensor $[\log 11]$

$\sigma_{v}^{2}=\frac{\left(\sigma_{x x}-\sigma_{y y}\right)^{2}+\left(\sigma_{y y}-\sigma_{z z}\right)^{2}+\left(\sigma_{z z}-\sigma_{x x}\right)^{2}+6\left(\tau_{x y}^{2}+\tau_{y z}^{2}+\tau_{z x}^{2}\right)}{2}$,

where the components $\sigma_{x x}, \sigma_{y y}, \sigma_{z z}$ are orthogonal normal stresses, and $\tau_{x y}, \tau_{y z}, \tau_{x z}$ the orthogonal shear stresses.

\subsection{Rib Cross-section}

A wide range of profiles of cross-section for ribs have been applied in engineering. Among them, T-shaped cross-section is rather popular due to some practical advantages. T-section offers greater and significantly more resistance. And the material is present where it should be and in the right quantities [LS03]. This makes the rib more economical and lighter and in turn again making it even more economical. However, the commonly used right-angled T-section has a stress concentration on the corner which results in a localized increase in stress and causes cracks or even catastrophic failure [KB13]. Therefore, we use hyperellipse to approximate $\mathrm{T}$ shape and form a hyperelliptic T-section. The formulation of a hyperellipse with order $n(n>$ 2) can be formulated as follows

$$
\left|\frac{x}{a}\right|^{n}+\left|\frac{y}{b}\right|^{n}=1
$$

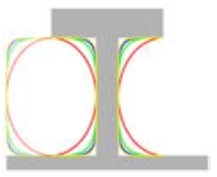

where $a$ and $b$ are semi-diameters. And as the order $n$ increases, the hyperellipse converges to a rectangle and the cross-section of rib tends to be a standard right-angled T-section as shown in the inset.
Hyperelliptic T-section inherits the advantages of usual T-section and improves stress concentration at the same time. To show stress distribution improvement of hyperelliptic T-section compared with right-angled one, we perform an FEA on the two types of ribs. We fix two ends of the rib, and impose loads on the middle. The results in Figure 7 indicate hyperelliptic T-section has better performance and can prevent from stress concentration to some extent.

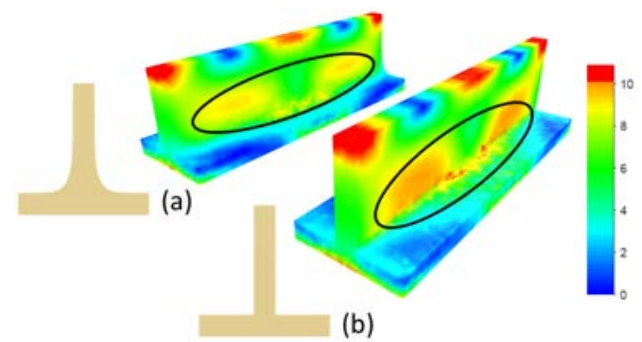

Figure 7: Hyperelliptic (a) and right-angled (b) T-section. Color encodes the von Mises stress. The stress of right-angled T-section in the black ellipse is much higher than that of hyperelliptic T-section.

\section{Rib Network Generation}

In this section, we will introduce the method to construct a rib network on arbitrary surface such that each rib aligns with the principal stress directions.

\subsection{Principal Stress Calculation}

Principal stresses are the components of a stress tensor when the basis is changed in such a way that the shear stress components become zero. Our rib network generation is based on principal stresses of the original shell structure, more precisely, placing ribs along principal stress directions. The stress tensor $\sigma$ has three real eigenvalues $\lambda_{1}, \lambda_{2}, \lambda_{3}$ and three mutually orthogonal eigenvectors $\mathbf{e}_{1}, \mathbf{e}_{2}, \mathbf{e}_{3}$, satisfying $\sigma \mathbf{e}_{i}=\lambda_{i} \mathbf{e}_{i}(i=1,2,3)$. Therefore, by multiplying a coordinate transformation matrix to transform the original coordinate system to coordinate system with axes $\mathbf{e}_{1}, \mathbf{e}_{2}, \mathbf{e}_{3}$, the stress tensor now only has three normal components, i.e., principal stresses and three eigenvectors principal stress directions.

Principal stress directions are useful as they indicate trajectories of internal forces and, therefore naturally encode the optimal topology for any structure for a given set of boundary condition$\mathrm{s}$ [ABVW14]. In our paper, we utilize principal stress directions to guide the construction of rib layout and align ribs with these directions. Therefore ribs can approximately best reinforce shell under external loads.

\subsection{Quad-mesh Extraction}

Using the principal stress field defined on the surface of input mesh, we calculate a global parametrization of the surface based on the MIQ [BZK09] method. With the parametrization result we extract a quad-mesh from the input surface using the method of QEx [EBCK13]. Since the edges of the resultant quad-mesh align with the principal stress directions of the surface, we can generate 
an initial rib network from the quad-mesh.

Principal Stress Field Smoothing First of all, we smooth the principal stress field to make it smoother for parametrization. The smoothness energy is defined based on the method in $[\mathrm{KN}$ P07], which blends a smoothness energy with an alignment term:

$$
E_{s m}=\lambda \sum_{e_{i j} \in E} \cos 4\left(\theta_{i}+r_{i j}-\theta_{j}+\alpha_{i}-\alpha_{j}\right)+(1-\lambda) \sum_{f_{i} \in F} \alpha_{i}^{2} .
$$

Here $\theta_{i}$ and $\theta_{j}$ are stress directions in local frames of face $f_{i}$ and $f_{j}$, respectively, and $r_{i j}$ is the angle between both local frames. The variables $\alpha_{i}$ and $\alpha_{j}$ are the direction deviations on face $f_{i}$ and $f_{j}$, respectively. The right most term is the penalty of large deviations which keeps the resulting cross field aligning with the original stress field. And factor $\lambda$ determines if there is more emphasis on smoothing or alignment, we set it to be 0.8 here. Figure 8 provides a comparison of stress field before and after smoothing.
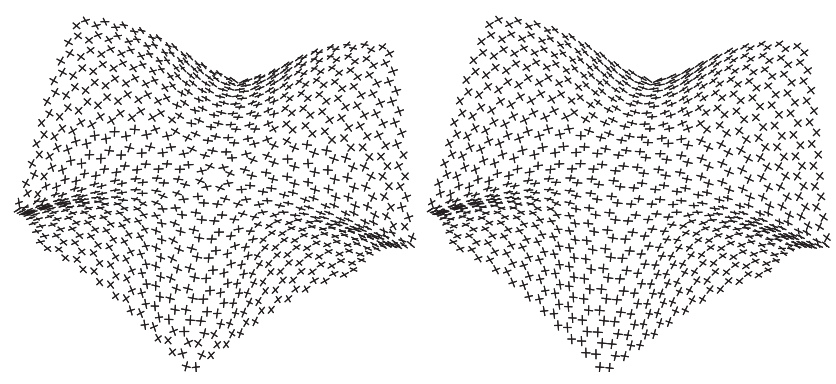

Figure 8: Left: principal stress directions. Right: principal stress directions after smoothing.

Global Parametrization In the global parametrization, we assign a $(u, v)$ parameter value to each vertex of the input mesh so that its iso-parameter lines on the surface are locally aligned with the principal stress field. We seek for the optimal parametrization by minimizing the following energy function

$$
E_{T}=\sum_{f_{i} \in F} A_{f_{i}}\left(\left\|\rho \nabla u-\mathbf{u}_{f_{i}}\right\|^{2}+\left\|\rho \nabla v-\mathbf{v}_{f_{i}}\right\|^{2}\right),
$$

where $\rho$ is a global scaling parameter which controls the length of edges in the resulting quad-mesh. And $\mathbf{u}_{f_{i}}$ and $\mathbf{v}_{f_{i}}$ are two orthogonal vectors in face $f_{i}$ corresponding to the cross field directions. The scalar $A_{f_{i}}$ is the area of face $f_{i}$.

Quad-mesh Extraction and Processing As long as we have the global parametrization result, a quad-mesh can be generated by just using the method of QEx [EBCK13]. We further use a simple Laplacian smoothing method to smooth the quad-mesh, and then project the smoothed quad-mesh to the original triangle mesh along vertex normal directions.

For some complex surfaces, the output quad-mesh may contain many singularities, which is harmful to the rib generation process. Therefore we use Peng's connectivity editing operations [PZKW11] to explicitly move and merge singularities.

\subsection{Rib Network Initialization}

Given a quad-mesh $\mathcal{M}(V, E, F)$, we trace its consecutive edges that don't bend too much and regard the resulting curve as a rib. Assume there is a rib $R$, we continue tracing and reach an adjacent edge $\mathbf{e}$ intersecting at point $\mathbf{p}$ with normal $\mathbf{n}$. To compute the angle between the rib $R$ and $\mathbf{e}$, we project edge $\hat{\mathbf{e}}$ adjacent to $\mathbf{e}$ in rib $R$ as well as edge $\mathbf{e}$ onto the tan-

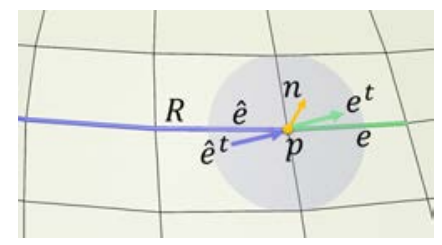
gent plane passing the point $\mathbf{p}$ perpendicular to the normal $\mathbf{n}$. Then the angle between the rib $R$ and edge $\mathbf{e}$ can be calculated by the two projected edge $\hat{\mathbf{e}}^{t}$ and $\mathbf{e}^{t}$ as follows

$$
\measuredangle(R, \mathbf{e})=\arccos \left(\frac{\hat{\mathbf{e}}^{t} \cdot \mathbf{e}^{t}}{\left\|\hat{\mathbf{e}}^{t}\right\|\left\|\mathbf{e}^{t}\right\|}\right),
$$

where $\hat{\mathbf{e}}^{t}=\hat{\mathbf{e}}-(\mathbf{n} \cdot \hat{\mathbf{e}}) \mathbf{n}$ and $\mathbf{e}^{t}=\mathbf{e}-(\mathbf{n} \cdot \mathbf{e}) \mathbf{n}$ are the projections of edges $\hat{\mathbf{e}}$ and $\mathbf{e}$ on the tangent plane, respectively.

When tracing a rib, a user-specified parameter $\phi$ is used to control the degree of bending. The algorithm to trace a rib is described in Algorithm 1.

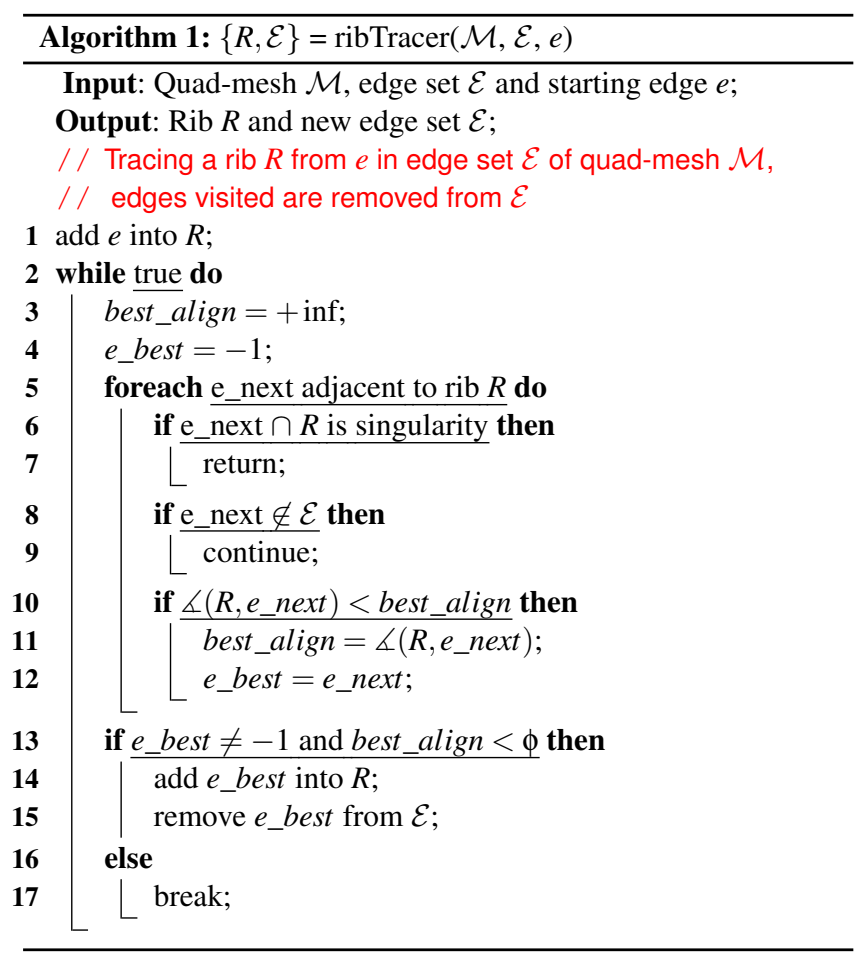

During the rib network tracing process, we first extract the treelike rib elements by tracing from the edges originated from the singularities of the quad-mesh. If a rib reaches a new singularity, we continue until the current tracing operation stops. After all the singularities are processed, namely, tree-like rib elements are all extracted, we trace from an arbitrary edge each time. When the rib meets with its starting point, we get a circular rib, otherwise, we get a curved rib. 
The complete rib network extraction process is described in Algorithm 2. Since the edges of quad-mesh align with the principal

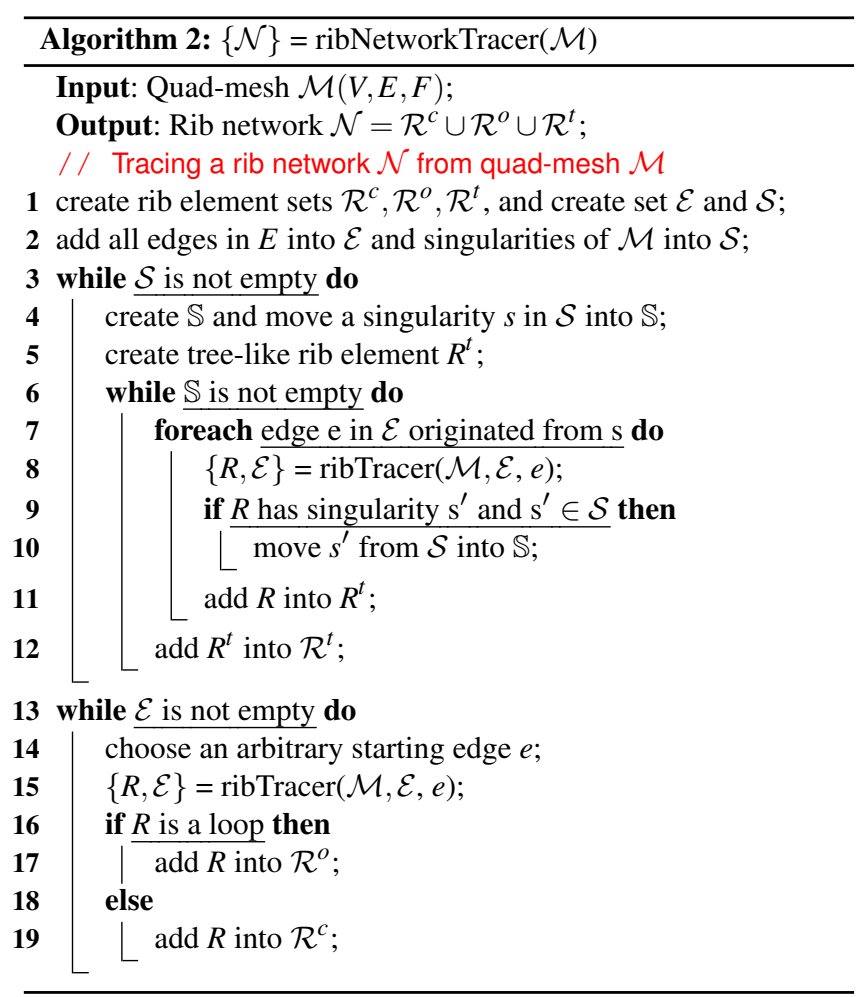

stress directions, the resultant rib network also lies along these directions. Once a dense rib network is obtained, we project all the ribs back to the input mesh to make them on the final shell.

\subsection{Rib Network Simplification}

In the previous step, we generate a dense rib network from quadmesh. But in fact, using all the ribs can be wasteful and unnecessary, and some ribs have little contribution to the shell. Moreover, since main deformations in shell structures are bending, a thick rib has much larger second moment of area than several thin ribs whose total cross-section area is same as the thick rib. Therefore, a sparse thick rib structure resists bending deformations more effectively than a dense thin rib structure. So we simplify the rib network to ensure that every rib is essential and obbligato. During rib simplification, we take each rib element as a whole to utilize the semantic information and retain structural integrity.

To measure the contribution of each rib element to the shell, we first calculate the total strain energy $U_{0}$ of the pure shell structure using $U_{0}=\mathbf{u}_{0}^{T} \mathbf{K}_{0}^{s}(t) \mathbf{u}_{0}$ with shell thickness $t$. Then given a certain volume $V$ of material, the strain energy of shell together with rib element $i$ is computed as

$$
U_{i}=\mathbf{u}_{i}^{T}\left(\mathbf{K}_{0}^{s}(t)+\mathbf{K}_{i}^{r}\left(w_{i}, h_{i}\right)\right) \mathbf{u}_{i} .
$$

Here $w_{i}$ and $h_{i}$ are the width and height of rib element. To make it simple, we assume the cross-sectional shape of all the ribs is square here. That is to say, $w_{i}=h_{i}$ and $l_{i} w_{i} h_{i}=V$ with total rib length $l_{i}$. And now the contribution for rib element $i$ is defined as

$$
C_{i}=1-\frac{U_{i}}{U_{0}}
$$

That means the more strain energy reduces, the more important the rib element is. We calculate the contribution values for all the rib elements, and discard any rib element whose contribution is less than a user-specified threshold value $\gamma$ at the same time. Figure 9 shows an example of rib network simplification.

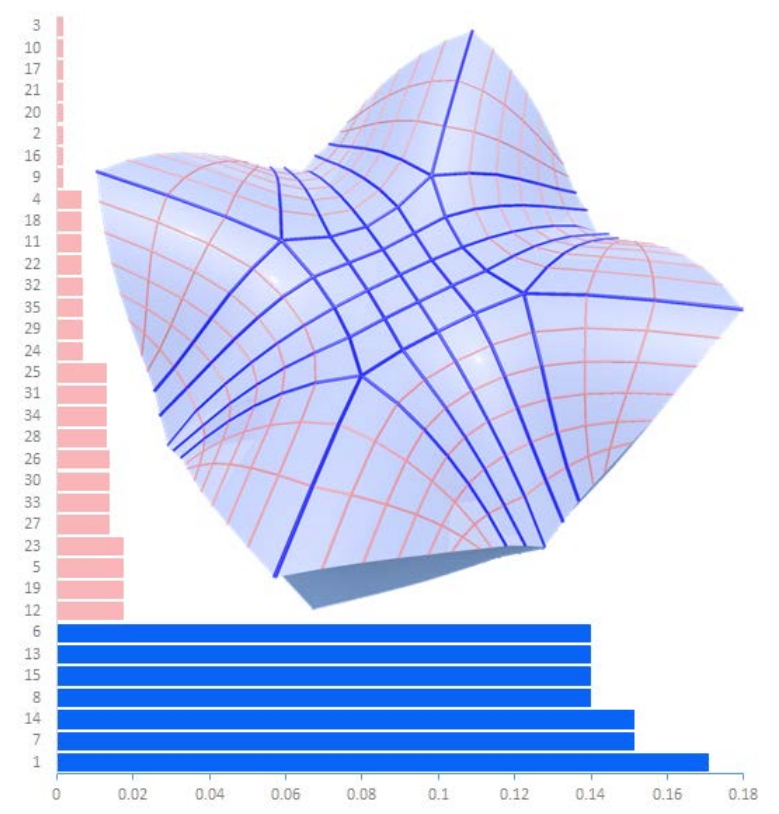

Figure 9: Rib network simplification of Guscio. The histogram on the left illustrates the contribution of each rib element. The resultant simplified rib network is colored in blue. Light orange rib elements are dropped.

Now we have an initial rib network, in the next section, we further describe the optimization of rib network which consists of rib flow optimization and rib cross-section optimization.

\section{Rib Network Optimization}

\subsection{Rib Flow Optimization}

In the previous section, we simplify a rib network generated from quad-mesh by dropping rib elements with little contribution. However, this removal operation affects the rest of ribs. We need to adjust the layout of rib network to achieve better distribution. Since our rib network is extracted from a quad-mesh $\mathcal{M}$, whose vertex positions are denoted as $\mathbf{p}_{v}^{0}, v=1,2, \cdots,|V|$, each rib point $R_{i j}$ has a unique integer $v_{i j}$ corresponding to a vertex in quad-mesh. And for each point $R_{i j}^{0}$ in a rib, we define its two moving directions as $\mathbf{d}_{i j}^{+}$and $\mathbf{d}_{i j}^{-}$. Assume each rib point has a signed moving distance $\delta_{i j}$ along its actual moving direction

$$
\mathbf{d}_{i j}=\left\{\begin{array}{ll}
\mathbf{d}_{i j}^{+} & \delta_{i j} \geqslant 0 \\
\mathbf{d}_{i j}^{-} & \delta_{i j}<0
\end{array} .\right.
$$


And then we can calculate the new position of each vertex of the quad-mesh by the following equation

$$
\mathbf{p}_{v}=\mathbf{p}_{v}^{0}+\sum_{v_{i j}=v}\left|\delta_{i j}\right| \mathbf{d}_{i j}
$$

We update the rib point position by $R_{i j}=\mathbf{p}_{v_{i j}}$ to keep the correspondence of ribs and quad-mesh. And the goal of rib flow optimization is to minimize the total strain energy of rib-shell structure by optimizing the position of each rib point, which is dependent on the moving distance $\delta_{i j}$.

Rib Moving Directions Basically, we hope all ribs to move along the directions orthogonal to itself. Considering most rib points in quad-mesh have a degree of four, for those points, we can define the moving directions as the two outgoing edge vectors with unit length, as shown in Figure 10. And the maximum moving distance for each direction is defined as the half of corresponding edge length $L_{i j}^{+}$and $L_{i j}^{-}$. For the rib point corresponding to singularity of

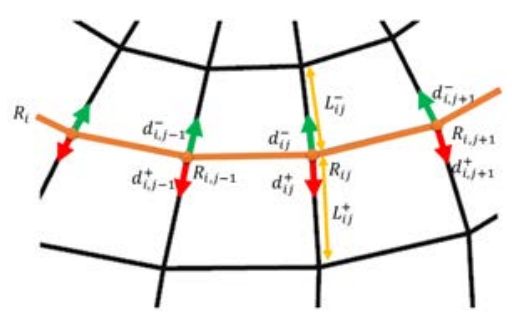

Figure 10: Moving directions for rib point with degree 4.

quad-mesh whose degree is not four, the moving direction is ambiguous to define. We take an alternative way and calculate its relative coordinates from a linear combination of its neighboring vertex positions, and update its position using this relative coordinates from its neighbors.

Problem Formulation The rib flow optimization is aimed at minimizing the total strain energy by changing the rib point positions. To keep the smoothness of ribs after flowing, we add a smoothing term by modifying the standard mid-point scheme (e.g., $\mathbf{p}_{i-1}+\mathbf{p}_{i+1} \approx 2 \mathbf{p}_{i}$ ) so that it reflects the varying density of rib points. Specifically, we define the smoothness of rib $R_{i}$ as

$$
S_{i}=\sum_{j=2}^{n_{i}-1}\left\|\left(1-u_{j}\right) R_{i, j-1}+u_{j} R_{i, j+1}-R_{i j}\right\|_{2}^{2},
$$

and the weight is define as

$$
u_{j}=\frac{\left\|R_{i j}^{0}-R_{i, j-1}^{0}\right\|_{2}}{\left\|R_{i j}^{0}-R_{i, j-1}^{0}\right\|_{2}+\left\|R_{i, j+1}^{0}-R_{i, j}^{0}\right\|_{2}} .
$$

Hence the objective function of rib flow optimization can be written as follows:

$$
\min _{\delta_{i j}} \frac{1}{2} \mathbf{u}^{T} \mathbf{K}\left(\delta_{i j}\right) \mathbf{u}+\lambda^{r} \sum_{i=1}^{n} S_{i}
$$

Here $\lambda^{r}$ is the weight of smoothing term and is set as 30 in our experiment. Since we only pursuit minor changes of rib point positions, we need to constrain their moving distances. When computing the rib moving directions, we can get the corresponding edge lengths. Thus we can bound the actual moving distance by

$$
-\frac{L_{i j}^{-}}{2} \leq \delta_{i j} \leq \frac{L_{i j}^{+}}{2} .
$$

And this constraint can further avoid the self-intersection of the final ribs. Figure 11 gives the results of rib flow optimization. And the strain energies of the shell for Guscio and Botanic reduced about $1.32 \%$ and $3.57 \%$, respectively.

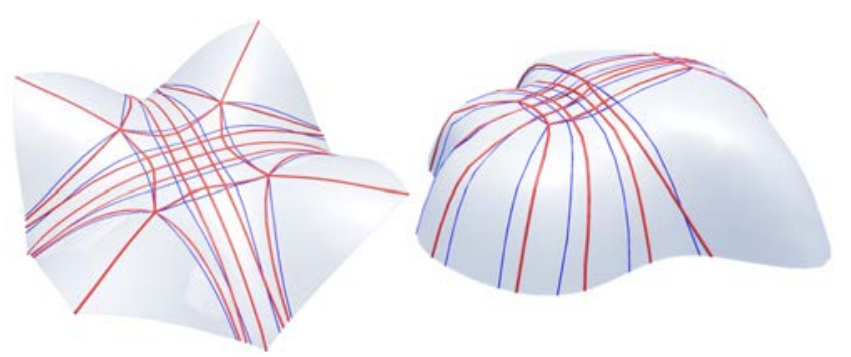

Figure 11: Rib network before (blue) and after (red) rib flow optimization for Guscio (left) and Botanic (right).

\subsection{Cross-Section Optimization}

The aim of rib cross-section optimization is to minimize the material usage of ribs while guaranteeing the overall physical performance of rib-shell structure. As illustrated before, hyperelliptic Tsection has many advantages. However, this non-linear optimization problem is very hard to solve and the initial values of all the parameters are difficult to specify. To tackle this problem, we first regard the cross-section as a rectangle, and optimize the rib size, i.e., width and height, to ensure the overall structure stays in a safe state by constraining the maximal stress. Then based on the rectangular ribs, we further optimize the cross-sectional shape to form a hyperelliptic T-section. And during the shape optimization, we choose an alternate easier but effective way which prevents the second moment of area from decreasing to guarantee the stiffness and strength of rib-shell structure.

\subsubsection{Size Optimization}

We assume the cross-sectional shape of each rib is rectangle first, and for rib point $R_{i j}$ the width and height are $w_{i j}$ and $h_{i j}$, respectively. Our goal is to minimize the rib volume while achieving certain stiffness requirement. The objective function is a combination of total rib volume and a smoothing energy. The smoothing term $S_{i}^{w}$ and $S_{i}^{h}$ are Laplacian smoothing of width and height along the ribs, and $\lambda^{w}$ and $\lambda^{h}$ are their weights, respectively. And the formulation of rib size optimization is as follows:

$$
\min _{\left\{w_{i j}, h_{i j}\right\}} \sum_{i=1}^{n}\left(V_{i}+\lambda^{w} S_{i}^{w}+\lambda^{h} S_{i}^{h}\right)
$$

$$
\text { s.t. }\left\{\begin{array}{l}
\sigma_{\max } \leq \bar{\sigma} \\
\underline{s} \leq w_{i j} \leq \bar{s}, \\
\underline{s} \leq h_{i j} \leq \bar{s} \\
\frac{1}{r} \leq \frac{w_{i j}}{h_{i j}} \leq r
\end{array}\right.
$$


Here $\sigma_{\max }$ is the maximum von Mises stress, and $\bar{\sigma}$ is the maximum allowable stress which reflects the strength limit of material. $\underline{s}$ and $\bar{s}$ are the lower and upper bound of rib size. And the last constraint keeps the ratio of width and height staying in a reasonable range. This optimization problem calculates the minimum needed material to keep the shell in a safe state.

\subsubsection{Shape Optimization}

Based on the rectangular ribs above, we further optimize the crosssectional shape to form a T-section.

Second Moment of Area The resistance of bending for a rib can be evaluated using second moment of area:

$$
\begin{aligned}
& I_{x}=\iint_{A} y^{2} d A, \\
& I_{y}=\iint_{A} x^{2} d A,
\end{aligned}
$$

where $A$ is cross-sectional domain we concern. Equation 16 and 17 indicate that for a shape with a given area, the more geometry accumulates away from $z$-axis the larger bending resistance it possesses. This provides a more convincing reason to use T-section from the perspective of mechanics principle.

Hyperelliptic T-Section We introduce here how to form a hyperelliptic T-section from a rectangular rib. We remove two halves of a hyperellipse from a rectangle as shown in the Figure 12. So the area of the cross-section is area of rectangle minuses the area of a hyperellipse. And the area inside the hyperellipse can be explicitly expressed in terms of the gamma function [Ros00].

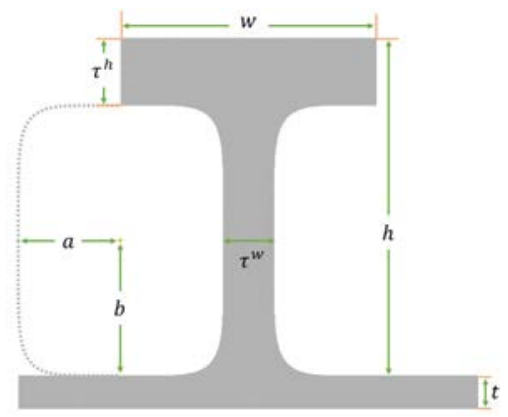

Figure 12: Hyperelliptic T-Section attached to a shell with thickness $t$.

Shape Optimization The goal of cross-sectional shape optimization is to further reduce material usage from a rectangular rib while maintaining the mechanical stiffness of the rib-shell structure under external load. So the objective function is also the volume of ribs

$$
\min \sum_{i=1}^{n} V_{i}\left(w_{i j}, h_{i j}, a_{i j}, b_{i j}\right),
$$

where $V_{i}\left(w_{i j}, h_{i j}, a_{i j}, b_{i j}\right)$ is the volume of rib $i$ with hyperelliptic T-section. $w_{i j}$ and $h_{i j}$ are width and height of the bounding box of cross-section, which we allow to stay near the original rectangular cross-section by the following constraints:

$$
\begin{aligned}
\left|w_{i j}-w_{i j}^{*}\right| & \leq \varepsilon_{w} w_{i j}^{*}, \\
\left|h_{i j}-h_{i j}^{*}\right| & \leq \varepsilon_{h} h_{i j}^{*} .
\end{aligned}
$$

Here $w_{i j}^{*}$ and $h_{i j}^{*}$ are width and height of rectangular rib from rib size optimization. $\varepsilon_{w}$ and $\varepsilon_{h}$ are parameters that control the change of rib width and height, respectively. And furthermore the parameters of hyperellipse are constrained by shell thickness $t$ as follows:

$$
\begin{aligned}
& w_{i j}=2 a_{i j}+\tau^{w}, \\
& h_{i j}=2 b_{i j}+\tau^{h},
\end{aligned}
$$

where $\tau^{w}$ and $\tau^{h}$ are flange and web thicknesses of the hyperelliptic T-section, respectively. To ensure the strength of the T-section, we further constrain $\tau^{w}$ and $\tau^{h}$ by the shell thickness $t$ using the following inequations:

$$
\begin{aligned}
& t<\tau^{w}<w_{i j}, \\
& t<\tau^{h}<h_{i j} .
\end{aligned}
$$

And the most important thing is that we must guarantee the mechanical performance of each rib. In the previous size optimization, we have forced the overall structure to stay in a safe state by constraining the maximal von Mises stress. However, tackling this constraint in a non-linear optimization is very time-consuming. So here we choose an alternate easier way, which prevents the second moment of area from decreasing, to ensure the mechanical performance of each rib. That is to say, the second moment of area of rib $i$ can't be less than original rib with rectangular cross-section.

$$
\begin{aligned}
& I_{x}(i) \geq I_{x}^{*}(i), \\
& I_{y}(i) \geq I_{y}^{*}(i) .
\end{aligned}
$$

Here $I_{x}^{*}(i)$ and $I_{y}^{*}(i)$ are the second moment of area for original rectangular rib $i$. And $I_{x}(i)$ and $I_{y}(i)$ are the second moment of area for hyperelliptic T-sectional rib $i$.

From the analysis above, the cross-sectional shape optimization can be formulated as a nonlinear optimization problem with objective function (Equation 18) and constraints (Equations 19, 20, 21 and 22).

\section{Results}

We have implemented our algorithm on a desktop PC with an Intel I5 CPU and 8G memory. The FEA and initial rib network generation process are implemented in $\mathrm{C}++$, while the rib optimization process is implemented with Matlab. The non-linear optimization problems (Equations 13, 15, 18) can be solved using the Interior Point Method [NW06] where the gradients are estimated using finite differences.

Parameters Setting In our experiment, the models are all scaled such that the maximum edge length of bounding box is 100 . we set the shell thickness $t$ as 1.2. The lower and upper bound of rib size are set as 0.8 and 8 , respectively. The maximum ratio of rib width and height $r$ is set as 4 . Note that the unit is millimeter throughout the paper. The scaling parameter $\rho$ for MIQ is between 18 and 30 in our method. And smoothing weights $\lambda^{w}$ and $\lambda^{h}$ are both set as 10. And parameters $\varepsilon^{w}$ and $\varepsilon^{h}$ are set as 0.2 here. The maximum allowable von Mises stress $\bar{\sigma}$ is set as 40 according to the yield strength of plastic (42 MPa). And the angle deviation threshold $\phi$ in Algorithm 1 is set as $\pi / 3$. The external loads are imposed as gravity by default. 


\subsection{Discussions}

Choice of Hyperelliptic Order As the order of hyperellipse in Equation 3 increases, it looks apparently like a rectangle with rounded corners. We calculate the performance of hyperelliptic T-section with different orders as shown in Figure 13. In the figure, we only show moments for bending resistance in height direction as they are more important than moments in width direction for shell structures. It can been seen that with the growth of orders, both values of hyperelliptic T-section are getting closer to corresponding values of right-angled T cross-section. But the computational cost of hyperelliptic shape rises as the increase of orders. Considering the time cost, we simply choose the order to be 6 where the changes of area and moment properties are small and the order is not too large.

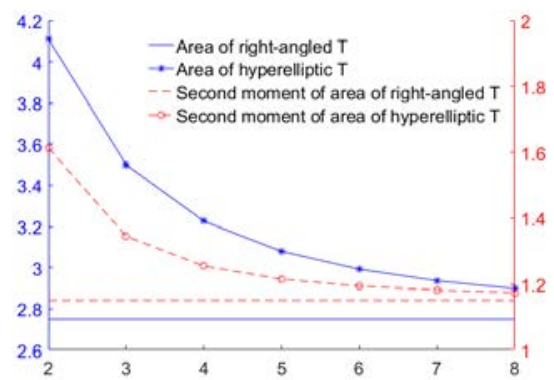

Figure 13: Area (blue) and second area moments (red) about orders of right-angled and hyperelliptic T-sections.

Iteratively Alternate Optimization of Rib Flow and Crosssection Actually, the rib flow optimization and cross-section optimization can be performed alternately and iteratively. And we have organized our code to jointly optimize locations and cross-sectional shapes of ribs. However, after a round of rib flow and cross-section optimization, the effect of rib flow in the next step is pretty minor. As a consequence, we abandon this strategy which seems to be more reasonable.

Choice of Mechanical Criterions From a purely superficial level, we uses different criterions at each stage. However, our method moves toward only one goal, namely, ensuring the structural safety with minimum material usage. Since the calculation of strain energy has a lower computational complexity than stress, we first pursue to compute a good distribution of ribs to reduce the total strain energy from a global perspective. But this is not enough, we need to further ensure the safety of the whole structure with minimum material. And hence we use von Mises stress and constrain it under the yield strength. But this non-linear cross-sectional shape optimization with a stress constraint is rather difficult to solve and the initial values are hard to choose. So we divide this cross-section optimization into size and shape optimization. During size optimization, we regard the cross-sectional shape as rectangle, and use stress constraint to ensure the safety. And then in the shape optimization, we optimize the superelliptic shape based on the previous rectangular ribs, while preventing the second moment of area which is easier to compute from decreasing to ensure the structural stiffness. In summary, our method takes some simple while accurate steps to solve a difficult and complex problems.

\subsection{Comparisons}

Comparison Between Concentrated and Distributed Load Our final rib-shell structure depends on the principal stress directions which are determined by external load conditions used in FEM. We compared the results under distributed and concentrated loads as shown in Figure 14, and found that under the concentrated external loads, ribs tend to be denser near the region of loading nodes.

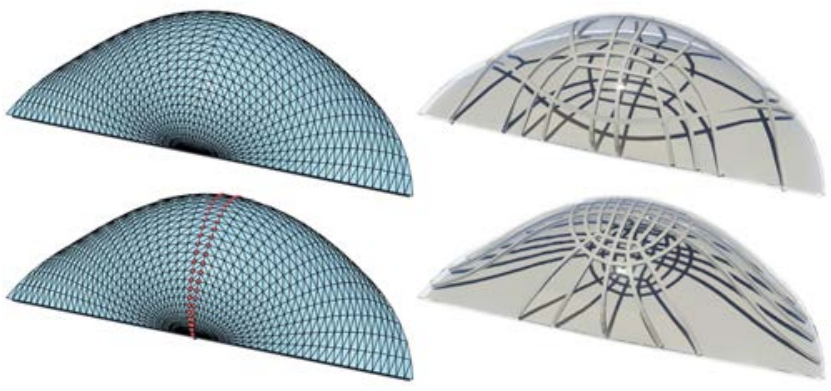

Figure 14: Top: distributed loads (gravity). Bottom: concentrated loads (load nodes are marked as red).

Comparison to Grid-shell Structure We presented the results of Voronoi grid-shell [PTP*15] and self-support quad grid-shell [VHWP12] structures as shown in Figure 15 for comparison. We regard the edges of Voronoi and quad grids as ribs with square crosssection. For fair comparison we keep the shell thickness and total volume of ribs as the same for the three types of structures. And the maximum stresses for our rib network, self-support quad and Voronoi grid structures are $39.99,38.37$ and 43.69 , respectively. Our rib network is comparable to the other two structures, while contains much simpler construction and fewer joints.

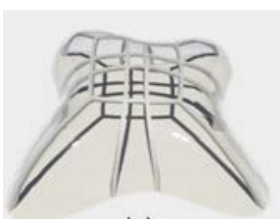

(a)

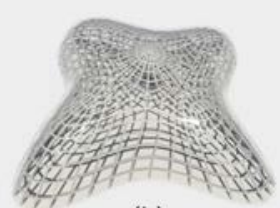

(b)

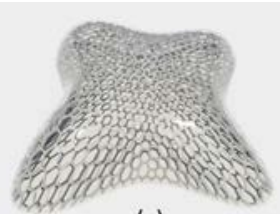

(c)
Figure 15: Results of Lilium. From (a) to (c): Our result, selfsupport quad shell [VHWP12] and Voronoi grid-shell [PTP*15].

\subsection{Results}

Examples Figure 16 showcases various rib-shell structures produced by our method. In Figure 17, we show the improvement of structural strength for the Lilium. To make this comparison fair, we reset the thickness of the shell such that it has the same volume as the final rib-reinforced shell. The maximum von Mises stress for the pure shell is $69.42 \mathrm{MPa}$ which is way beyond the allowable yield strength, and it reduces below $40 \mathrm{MPa}$ for the rib-reinforced shell. This comparison indicates rib-reinforced shell structures have a much higher strength than the pure shells with the same amount of material. 


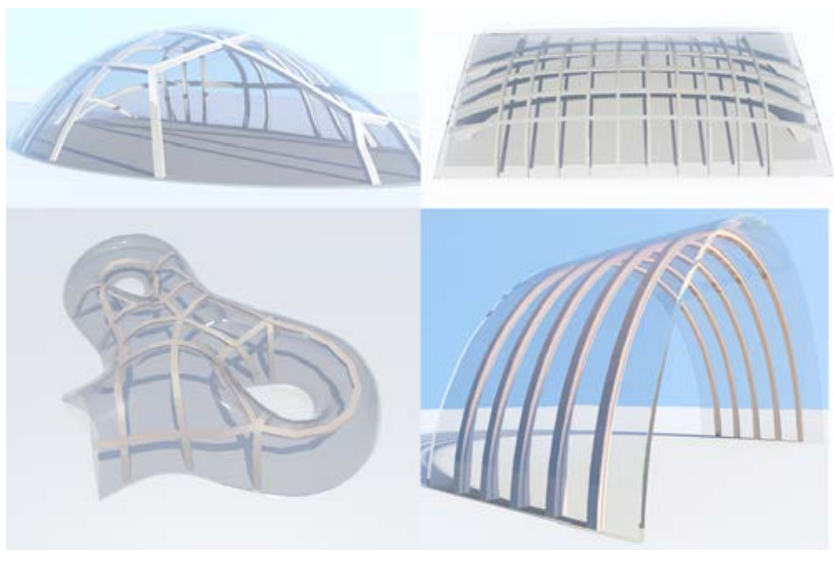

Figure 16: Rib-shell structures generated by our system, including Snail, Neumunster, Aquadom, and Bridge.
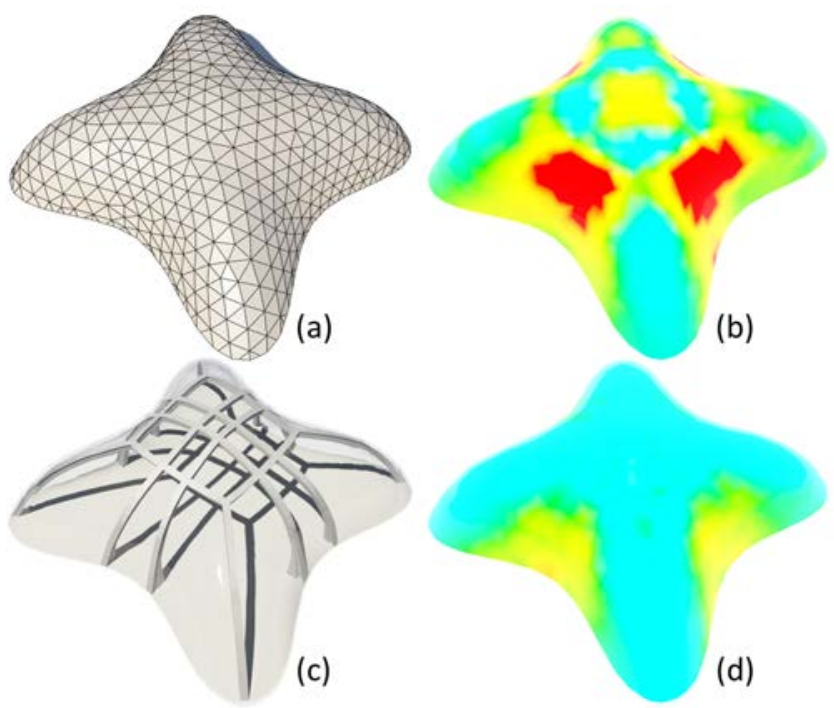

Figure 17: Rib-reinforced shell structure (c) generated from input mesh (a). And (b) and (d) are coloring visualizations of von Mises stress for pure shell and rib-reinforced shell, respectively, which cost the same volume of material. The colorbar is the same as Figure 1 .

Statistics Table 1 summarizes the statistics of the results shown in the paper. The most time-consuming part of initial rib network generation process lies on the MIQ parametrization, which depends on the number of faces and principal stress field singularities. And the rib network optimization takes a few minutes to dozens of minutes. Since rib size optimization problem involves the constraint of von Mises stress which makes this optimization particularly timeconsuming. And in the cross-sectional shape optimization problem, we take the second moment of area as constraint, which is smoother and easier to solve. So running times reduce a lot.

Physical Experiments After we get the final rib network, we perform a boolean operation on ribs and shell and get a watertight
Table 1: Statistics of applying our algorithm to various models shown in the paper.

\begin{tabular}{c|c|c|c|c|c}
\hline \multirow{2}{*}{ Models } & \multirow{2}{*}{$\begin{array}{l}\text { Mesh } \\
\text { V /F }\end{array}$} & \multicolumn{4}{|c}{ Times } \\
\cline { 3 - 6 } & $441 / 800$ & 0.71 & 7.7403 & 27.4322 & 1.7985 \\
\hline Guscio & $441 /(\mathrm{m})$ & FlowOpt(m) & SizeOpt(m) & ShapeOpt(m) \\
\hline Snail $^{1}$ & $871 / 1653$ & 3.48 & 3.2187 & 16.3718 & 1.6766 \\
\hline Snail $^{2}$ & $871 / 1653$ & 4.61 & 7.4263 & 32.2148 & 2.3598 \\
\hline Aquadom & $1078 / 2001$ & 14.13 & 4.5472 & 24.7954 & 3.1603 \\
\hline Bridge & $998 / 1851$ & 11.65 & 2.8563 & 5.5341 & 0.7336 \\
\hline Lilium & $559 / 1030$ & 1.30 & 4.4815 & 42.3216 & 0.7056 \\
\hline Neumunster & $220 / 380$ & 0.14 & 2.4154 & 25.7607 & 1.7236 \\
\hline Leaf & $1500 / 2840$ & 24.90 & 0.9927 & 5.2425 & 0.5428 \\
\hline Kite & $1571 / 2918$ & 27.58 & 4.1305 & 35.0450 & 0.9897 \\
\hline
\end{tabular}

Note: The unit for Init is second, while the unit for FlowOpt, SizeOpt and ShapeOpt is minute.

${ }^{1}$ Distributed loads imposed, see the top of Figure 14.

${ }^{2}$ Concentrated loads imposed, see the bottom of Figure 14.

mesh. And we fabricated rib-shell structure of Guscio by an SLA 3D printer as shown in Figure 18. To show the effect of ribs, we

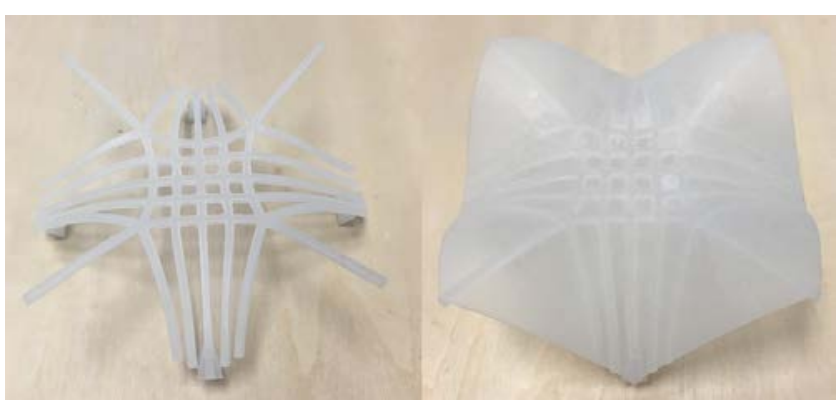

Figure 18: Left: rib network. Right: shell reinforced by ribs.

printed a shell which costs the same amount of material. As shown in Figure 19 (a-1) and (b-1), the weight of the pure shell is $19.26 \mathrm{~g}$, while the weight of the shell reinforced by ribs is $19.25 \mathrm{~g}$. To compare the stiffness of the two models, we impose external loads on the center as shown in the bottom of Figure 19 to see the deformation. Figure $19(\mathrm{a}-2)$ and $(\mathrm{b}-2)$ are the initial states of the two shells for reference, and (a-3) and (b-3) are the final state under external loads. In Figure 19 (a-3), the pure shell is under a $30 \mathrm{~N}$ load, the displacement at the center is $5.6 \mathrm{~mm}$. While for the shell reinforced by ribs, the displacement at the center is $1.2 \mathrm{~mm}$ under a $33 \mathrm{~N}$ load. Thus our rib-shell structure achieves over 4 times higher strength compared with a pure shell under the same external load with the same volume of materials.

Extensions Our system can also generate other results similar to rib-shell structures. From the images of kite and leaf shown in Figure 20 (a) and (c), we generate the triangular meshes of the two models. Then we set the boundary conditions and external loads. For the kite model, the center is fixed, and loads are imposed to every node to simulate wind force. And the for the leaf model, the petiole is fixed and gravity is applied. The resultant rib-shell structures for kite and leaf generated by our system are shown in Figure 20 (b) and (d), which seem to make sense to some extent.

\section{Conclusions}

In this paper, we present a practical framework that allows to generate and optimize a rib network to reinforce the structural stiffness 


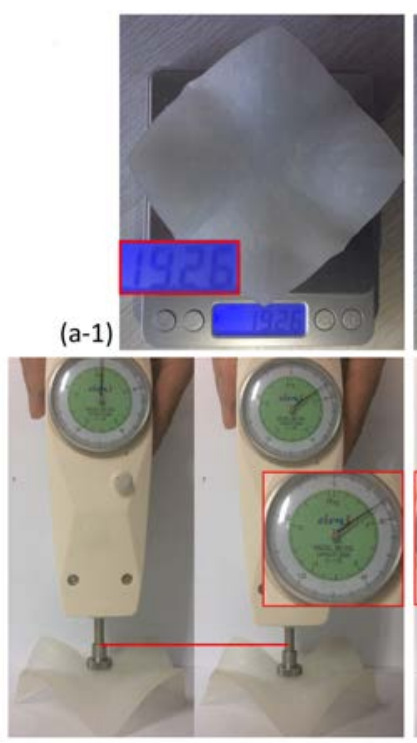

$(a-2)$
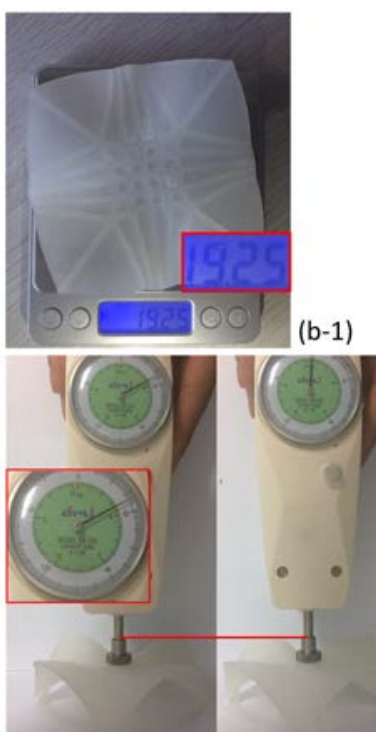

(b-3)

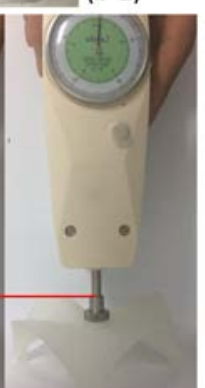

(b-2)

Figure 19: The fabricated shell (a-1) and rib-reinforced shell (b-1) cost about the same amount of materials (19.26g for the former and $19.25 \mathrm{~g}$ for the latter). When imposing loads at the center, the pure shell has a displacement of $5.6 \mathrm{~mm}$ under a $30 \mathrm{~N}$ load (a-3), while the displacement is $1.2 \mathrm{~mm}$ for the rib-reinforced shell under a $33 \mathrm{~N}$ load (b-3). And (a-2) and (b-2) are initial states without load for reference. The results of physical experiment agree with the FEA results as shown in Figure 1.
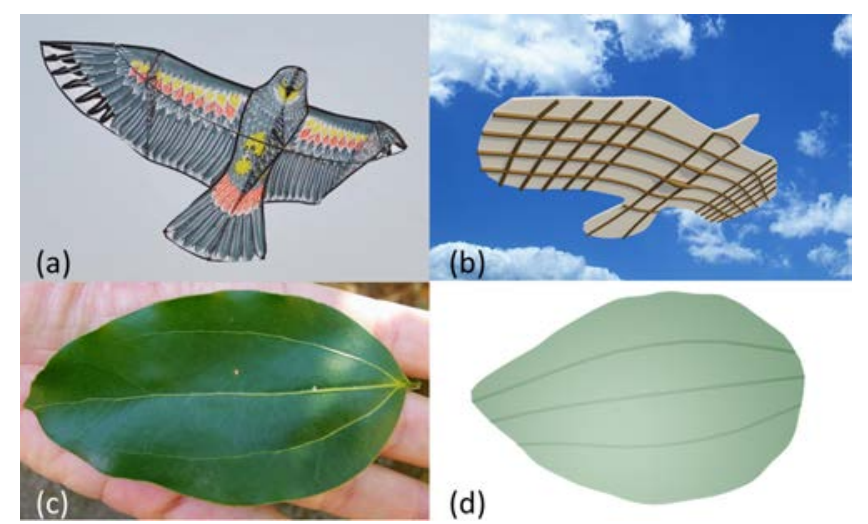

Figure 20: Rib-shell structures for kite (b) and leaf (d) models generated by our system, which imitate the things ( $a$ and $c$ ) in real world to some extent.

and physical strength of input shell structure. Our basic idea is to place ribs along the principal stress directions which are calculated from FEA results and encode the optimal topology. A variety of examples validate the capability and efficiency of the computational framework.

Limitations Our method is based on the principal stress directions which are computed from FEA results. However, the FEA results depend on the external loads. As a consequence, our rib-shell struc-

ture has remarkable structural properties under the initial external load. When the loads change, the overall performance can not be guaranteed. Actually, this is a common limitation for all FEAbased method. Besides, we ignore aesthetics during the generation and optimization of rib network, which we plan to tackle as a future work. However, for low-level aesthetic standard like symmetry, our method can generate symmetric results as long as the input surface as well as the external loads and boundary conditions for FEM are symmetric, like the examples in Figure 9.

Future Works In the future, we will investigate the relation between principal curvature directions and principal stress directions which are both cross vector fields defined on a surface. Since the former reflects geometric property and appearance, but the latter involves in mechanical performance. Study on combination of the two fields will be an interesting thing.

\section{Acknowledgments}

We would first like to thank the reviewers for the valuable comments. Also, we would especially like to thank Chi-Han Peng and Peter Wonka for providing the code of [PZKW11]. This work is supported by the National Natural Science Foundation of China (61672482, 11626253), the One Hundred Talent Project of the Chinese Academy of Sciences, EU Horizon 2020 RISE 2017 PDE-GIR project 778035, the FP7 European project Dr Inventor FP7-611383, EU H2020 funded project AniAge (H2020-MSCA-RISE-691215) and EU FP7 funded project AniNex (FP7-IRSES-612627).

\section{References}

[ABVW14] Adriaenssens S., Block P., Veenendaal D., WILliams C.: Shell structures for architecture: form finding and optimization. Routledge, 2014. 4,5

[ACTR04] Ansola R., Canales J., Tarrago J., Rasmussen J.: Combined shape and reinforcement layout optimization of shell structures. Structural and Multidisciplinary Optimization 27, 4 (2004), 219227. 3

[ARR12] ARASHMEHR J., RAHIMI G., RASOULI S.: Numerical and experimental stress analysis of stiffened cylindrical composite shell under transverse end load. Journal of World Academy of Science, Engineering and Technology 67 (2012), 1298-1302. 2, 3

[BZK09] Bommes D., Zimmer H., Kobbelt L.: Mixed-integer quadrangulation. ACM TRANS. GRAPH (2009), 1-10. 5

[CK13] ChANG Y. Z., KANG Y. G.: Dynamic stability analysis of steel concrete composite of ribbed shell under stepped loads. In Advanced Materials Research (2013), vol. 712, Trans Tech Publ, pp. 815-821. 2,3

[CL97] Chung J., LeE K.: Optimal design of rib structures using the topology optimization technique. Proceedings of the Institution of Mechanical Engineers, Part C: Journal of Mechanical Engineering Science 211, 6 (1997), 425-437. 3

[CW07] CutLER B., Whiting E.: Constrained planar remeshing for architecture. In Proceedings of Graphics Interface 2007 (2007), ACM, pp. 11-18. 2

[DB11] Davis L. K., BLOCK P.: Scaffolding to structure: Construction in thin-shell masonry. 2

[DJMH13] Ding X., Ji X., MA M., Hou J.: Key techniques and applications of adaptive growth method for stiffener layout design of plates and shells. Chinese Journal of Mechanical Engineering 26, 6 (2013), $1138-1148.2,3$ 
[EBCK13] EbKe H.-C., Bommes D., CAMPen M., Kobbelt L. QEx: Robust quad mesh extraction. ACM Trans. Graph. 32, 6 (Nov. 2013), 168:1-168:10. 5, 6

[EDE10] Esterhuizen G. S., Dolinar D. R., Ellenberger J. L. Pillar and roof span design in stone mines. Extracting the Science A Century of Mining Research (2010). 2

[EKS*10] Eigensatz M., Kilian M., Schiftner A., Mitra N. J., Pottmann H., PAuly M.: Paneling architectural freeform surfaces. ACM transactions on graphics (TOG) 29, 4 (2010), 45. 2

[JDX14] Ji J., DING X., XIONG M.: Optimal stiffener layout of plate/shell structures by bionic growth method. Comput. Struct. 135 (Apr. 2014), 88-99. 3

[Joh58] JohannES M.: Stability of rib-reinforced cylindrical shells under lateral pressure. IABSE 18 (1958). 3

[KB13] KoKCHAROV I., BUROV A.: Structural integrity analysis. Switzerland: Geneva (2013). 5

[KLC16] KWOK T.-H., LI Y., CHEN Y.: A structural topology design method based on principal stress line. Computer-Aided Design 80 (2016), 19-31. 2, 3

[KNP07] KÄLBERER F., NiEser M., POlthier K.: Quadcover - surface parameterization using branched coverings. Comput. Graph. Forum 26, 3 (2007), 375-384. 6

[LLW15] LI Y., LIU Y., WANG W.: Planar hexagonal meshing for architecture. IEEE transactions on visualization and computer graphics 21, 1 (2015), 95-106. 2

[Log11] LogAN D. L.: A first course in the finite element method. Cengage Learning, 2011. 5

[LS03] LAM Y., SANThIKUMAR S.: Automated rib location and optimization for plate structures. Structural and multidisciplinary optimization 25, 1 (2003), 35-45. 2, 3, 5

[LS14] LIU Y., ShIMOdA M.: Parameter-free optimum design method of stiffeners on thin-walled structures. Structural and Multidisciplinary Optimization 49, 1 (2014), 39-47. 3

[LSZ*14] Lu L., Sharf A., Zhao H., Wei Y., Fan Q., Chen X. Savoye Y., Tu C., Cohen-Or D., Chen B.: Build-to-last: Strength to weight 3d printed objects. ACM Trans. Graph. 33, 4 (July 2014), 97:1-97:10. 3

[LXW*11] LiU Y., Xu W., Wang J., Zhu L., Guo B., Chen F. WANG G.: General planar quadrilateral mesh design using conjugate direction field. ACM Trans. Graph. 30, 6 (Dec. 2011), 140:1-140:10. 2

[NW06] Nocedal J., Wright S.: Numerical optimization. Springer Science \& Business Media, 2006. 9

[PJH*15] Pottmann H., Jiang C., Höbinger M., Wang J., BomPAS P., WALlner J.: Cell packing structures. Comput. Aided Des. 60, C (Mar. 2015), 70-83. 2

[PS01] PRUsty B. G., SATSAngi S.: Analysis of stiffened shell for ships and ocean structures by finite element method. Ocean Engineering 28, 6 (2001), 621-638. 2, 3

[PSB*08] PotTmann H., Schiftner A., Bo P., Schmiedhofer H., WANG W., BALDASSINI N., WALLNER J.: Freeform surfaces from single curved panels. ACM Transactions on Graphics (TOG) 27, 3 (2008), 76. 2

[PTP*15] Pietroni N., Tonelli D., Puppo E., Froli M., ScopigNo R., Cignoni P.: Statics aware grid shells. Comput. Graph. Forum 34, 2 (May 2015), 627-641. 2, 10

[PZKW11] Peng C.-H., Zhang E., Kobayashi Y., Wonka P.: Connectivity editing for quadrilateral meshes. ACM Trans. Graph. 30, 6 (Dec. 2011), 141:1-141:12. 6, 12

[Ros00] Rosin P. L.: Fitting superellipses. IEEE Transactions on Pattern Analysis \& Machine Intelligence 22, 7 (2000), 726-732. 9

[SB10] SCHIFTNER A., BALZER J.: Statics-sensitive layout of planar quadrilateral meshes. Advances in Architectural Geometry (2010), 221236. 2

(c) 2017 The Author(s)

Computer Graphics Forum (c) 2017 The Eurographics Association and John Wiley \& Sons Ltd
[SB12] SzCZEPANIK M., BURCZYŃSKI T: Swarm optimization of stiffeners locations in 2-d structures. Bulletin of the Polish Academy of Sciences: Technical Sciences 60, 2 (2012), 241-246. 3

[SHWP09] Schiftner A., HöBinger M., WAllner J., PotTMAnN H.: Packing circles and spheres on surfaces. ACM Trans. Graph. 28, 5 (Dec. 2009), 139:1-139:8. 2

[SZXG14] Su L., ZhU S.-L., XIAO N., GaO B.-Q.: An automatic grid generation approach over free-form surface for architectural design. Journal of Central South University 21, 6 (2014), 2444-2453. 3

[TFCM16] TaM K. M. M., Fine N., Coleman J., Mueller C: Stress line additive manufacturing (slam) for 2.5-d shells. Journal of the International Association for Shell and Spatial Structures (J. IASS) 57, 4 (2016). 2, 3

[TM15] TAM K., Mueller C.: Stress line generation for structurally performative architectural design. In Proceedings of the Association for Computer Aided Design in Architecture (ACADIA) Conference, Cincinnati, Ohio, US (2015). 3

[TSG* $\left.{ }^{*} 4\right]$ Tang C., Sun X., Gomes A., Wallner J., PottmanN H.: Form-finding with polyhedral meshes made simple. ACM Trans. Graph. 33, 4 (July 2014), 70:1-70:9. 2

[VHWP12] Vouga E., Höbinger M., Wallner J., Pottmann H. Design of self-supporting surfaces. ACM Transactions on Graphics (TOG) 31, 4 (2012), 87. 10

[VK01] Ventsel E., Krauthammer T.: Thin plates and shells: theory, analysis, and applications. CRC press, 2001. 2, 4

[WAWS17] Wu J., Aage N., Westermann R., Sigmund O.: Infill optimization for additive manufacturing-approaching bone-like porous structures. IEEE Transactions on Visualization and Computer Graphics (2017). 3

[WAZ17] WANG D., ABDALla M. M., ZHANG W.: Buckling optimization design of curved stiffeners for grid-stiffened composite structures. Composite Structures 159 (2017), 656-666. 3

[WWY*13] Wang W., Wang T. Y., Yang Z., LiU L., Tong X., TONG W., DENG J., CHEN F., LIU X.: Cost-effective printing of 3d objects with skin-frame structures. ACM Transactions on Graphics (Proc. SIGGRAPH Aisa) 32, 5 (2013), Article 177: 1-10. 2

[YYPM11] YANG Y.-L., YANG Y.-J., POTTMANN H., Mitra N. J.: Shape space exploration of constrained meshes. ACM Trans. Graph. 30, 6 (Dec. 2011), 124:1-124:12. 2

[ZKWG16] Zhou Y., Kalogerakis E., Wang R., Grosse I. R.: Direct shape optimization for strengthening $3 \mathrm{~d}$ printable objects. In Computer Graphics Forum (2016), vol. 35, Wiley Online Library, pp. 333-342. 3

[ZSW10] Zadravec M., Schiftner A., Wallner J.: Designing quad-dominant meshes with planar faces. Computer Graphics Forum 29, 5 (2010), 1671-1679. 2

[ZXZ*16] Zhao H., Xu W., Zhou K., Yang Y., Jin X., Wu H.: Stress-constrained thickness optimization for shell object fabrication. Computer Graphics Forum (2016). 3 